\title{
Generation of Multiple Weights in the Opportunistic Beamforming Systems
}

\author{
Guangyue LU ${ }^{1,2}$, Lei ZHANG ${ }^{2}$, Houquan YU ${ }^{1}$, Chao SHAO ${ }^{2}$ \\ ${ }^{1}$ Electronics and Information College, Yangtze University, Jingzhou, China \\ ${ }^{2}$ Department of Telecommunications Engineering, Xi'an Institute of Posts and Telecommunications, Xi'an, China \\ E-mail:tonylugy@yahoo.com,chaoshao@xupt.edu.cn \\ Received April 18, 2009; revised April 29, 2009; accepted May 31, 2009
}

\begin{abstract}
A new scheme to generate multiple weights used in opportunistic beamforming (OBF) system is proposed to deal with the performance degradation due to the fewer active users in the OBF system. In the proposed scheme, only two mini-slots are employed to create effective channels, while more channel candidates can be obtained via linearly combining the two effective channels obtained during the two mini-slots, thus increasing the multiuser diversity and the system throughputs. The simulation results verify the effectiveness of the proposed scheme.
\end{abstract}

Keywords: Opportunistic Beamforming (OBF), Multiuser Diversity, System Throughputs, Scheduling

\section{Introduction}

With the development of the wireless communication, increasing the spectrum efficiency and data rates is becoming the major task, especially in the downlink case. Multiple-Input-Multiple-Output (MIMO) technique [1] can improve the spectrum efficiency with no need of more bandwidth by employing multiple antennas at both transmitter and receiver. Therefore MIMO technique is becoming one of the most promising techniques in the future communication systems (e.g., LTE, B3G), and coherent beamforming [2] and dirty paper coding [3] are two ways to improving the spectrum efficiency. However the full channel information for all users at the transmitter is required to realize the coherent beamforming and dirty paper coding, which is not realistic with the increasing of the number of the users and antennas because of the waste of the systems resource to feedback the channel information from the receivers to the transmitter.

In wireless communication system, many users are communicating with the base station, and the system throughput can be improved by suitably scheduling (through, e.g., maximum throughput (MAX) scheduling algorithm or proportional fairness (PF) scheduling algorithm) the user with large channel gains to transmit its packets, which is known as the multiuser diversity
(MUD) [4]. In contrast to the channel equalization used in the traditional communication systems to combat the effect of the multipath fading channel on the data transmission, it is the channel fluctuations that is the source of the MUD and the MUD will be enlarged with the increase of the dynamic range of the channel fading. The larger the dynamic range of the channel fluctuations, the higher peak of the channels and the larger the multiuser diversity gain. Hence to achieve large MUD requires the large channel dynamic range and the suitable scheduling scheme.

However, the MUD gain will be limited by the small dynamic range of the channel fluctuations due to the availability of light-of-sight (LOS) path and little scatting in the environment and the slowly channel fading compared to the delay constraint of the services. Thus those users with small channel gain and fluctuations may not be scheduled to transmit their packets and their QoS can not be met.

In [5], random fading is induced purposely in multiple-input-single-output (MISO) systems when the environment has little scatting and/or the fading is slow to increase the MUD gain of the system by multiplying the transmit data with different weighting factors at each transmitting antenna. When the weighting factors are phase-conjugate with the independent channels from the user to the transmitting antennas, this user is in its 
beamforming configuration state and its channel peak values occur. When the number of the users in the systems is large enough, the probability that at lease one user is in its beamforming configuration state is large and the throughput of the system can approach that of the coherent beamforming with only partial channel information (i.e., the overall SNR) feedback. And the scheme in [5] is interpreted as the opportunistic beamforming (OBF).

However, one of the limits of the OBF is the requirement of large number of users in the system simultaneously and the system throughput will be degraded when the number of the users in the system is not too large. When fewer users are active in the system, the MISO system in [5,6] is extended to MIMO in [7], that is, multiple antennas are also employed at the receivers, which equivalently increase the number of visual active users and, thus, the system throughput. However, the feedback and the costs of each user will be inevitably increased with the increase of the number of the users and the employed receiving antennas. The weighting factors used at the transmitting antennas in [5] are totally random among different time slots. However, since the base station possesses all the users' channels information at current time slot and the previous time slots, the weighting factors can be generated in an pseudo-random manner, that is, the former weighting factors that create beamforming configuration state for one user can be used, in some way, to generate the current former weighting factors only if the coherent time of the channels is large enough $[8,9]$.

Since the random weighting factors strongly affect the channel states, multiple weighting vectors at several mini-slots in one time slot [10] are used to create multiple induced channels, and the one with larger channel gain is selected and the corresponding weighting vector is used as the current weighting vector. The OBF with multiple weighting factors (MW-OBF) can improve the throughput of OBF-CDMA systems. Since several mini-slot are used to 'train' the best weighting factors, some mini-slots and power resources are wasted in MW-OBF.

In [11], two multiple weight OBF schemes tailored for fast fading and slow fading scenarios respectively are investigated and the tight upper bounds of the data rates for both schemes are derived. It is claimed that the faster the fading is, the less weight vectors are desired; and the more users there are, the less weight vectors are desired. To overcome the problem of limited multiuser diversity in a small population, [12] devises a codebook-based OBF (COBF) technique, where the employed unitary matrix changes with time slot to induce larger and faster channel fluctuations in the static channel and to provide further selection diversity to the conventional OBF technique. Compared with [10], the COBF technique reduces the required number of mini-time slots, and, since it is the size of codebook, not the number of mini-time slots, that determines the amount of supplementary selection diversity, the system throughput can be increased without limitation from the number of mini-time slots. However, the receiver should estimate all of channels from it to the transmitters.

In this paper, a new scheme to generate multiple weights used in OBF is proposed to deal with the performance degradation due to the less number of users in the OBF system. In the proposed scheme, only the equivalent channels at two mini-slots are required to be estimated, as in the normal OBF. The paper is outlined as follow: after the introduction of conventional OBF and MW-OBF in Section 2, the proposed scheme with only two mini-slots to create more channel candidates via linearly combining the two effective channels at the receiver is developed and analyzed in Section 3. Section 4 gives the numerical results to verify the effectiveness of the proposed scheme from different aspects. The paper is concluded in Section 5.

\section{Conventional OBF and MW-OBF}

Assume there are $N$ transmitting antennas at the base station and one receiving antenna at each user side, the channel gain vector for the $k$-th user is $\boldsymbol{H}_{k}(t)$ $=\left[h_{1 k}(t), \ldots, h_{N k}(t)\right]^{T}$, where $h_{n k}(t)(n=1, \ldots, N)$ is the channel gain from the $n$-th antennas to the $k$-th user at time $t$. And the transmitting signal $x(t)$ is multiplied with the weight vector $\boldsymbol{V}(t)=\boldsymbol{e}_{\theta}^{T}(t) \boldsymbol{\alpha}(t)$, where $\boldsymbol{V}(t) \in C^{1 \times N}$, diagonal matrix $\boldsymbol{\alpha}(t)=\operatorname{diag}\left(\sqrt{\alpha_{1}(t)}, \ldots\right.$, $\left.\sqrt{\alpha_{N}(t)}\right)$ denotes the power allocation on each transmitting antenna, and $\boldsymbol{e}_{\theta}(t)=\left[e^{j \theta_{1}(t)}, \ldots, e^{j \theta_{N}(t)}\right]^{T}$ is random phase vector applied to the signal, $\theta_{n}(t)$ are the independent random variables uniformly distributed over $[0$, $2 \pi)$. In order to preserving the total power, $\sum_{n=1}^{N} \alpha_{n}(t)=1$, where random variable $\alpha_{n}(t)$ varies from 0 to 1 . Then the received signal for the $k$-th user is,

$$
\begin{aligned}
y_{k}(t) & =\sum_{n=1}^{N} \sqrt{\alpha_{n}(t)} e^{j \theta_{n}(t)} h_{n k}(t) x(t)+z_{k}(t) \\
& =\boldsymbol{e}_{\theta}^{T}(t) \boldsymbol{\alpha}(t) \boldsymbol{H}_{k}(t) x(t)+\boldsymbol{z}_{k}(t) \\
& \operatorname{def} \\
& =\tilde{H}_{k}(t) x(t)+\boldsymbol{z}_{k}(t)
\end{aligned}
$$

where $\tilde{H}_{k}(t)=\boldsymbol{e}_{\theta}^{T}(t) \alpha(t) \boldsymbol{H}_{k}(t)=\boldsymbol{V}(t) \boldsymbol{H}_{k}(t) \quad$ is the equivalent channel (i.e., overall channel) for user $k$, and $\boldsymbol{z}_{k}(t)$ be the independent and identically distributed AWGN. 
From (1), when $\boldsymbol{H}_{k}(t)$ are phase-conjugate with $\boldsymbol{e}_{\theta}(t)$, that is, $\theta_{n}(t)=-\operatorname{angle}\left(h_{n k}(t)\right) \quad(n=1, \ldots, N)$, $\tilde{H}_{k}(t)$ are the coherent sum of $h_{n k}(t)$, and user $k$ is in its beamforming configuration state. Thus large channel gain for user $k$ is obtainable.

In a heavy load system (i.e., the number of active user are large enough), by varying the weights $V(t)$, there is a large possibility that some users are in or nearly in their beamforming configuration states. Using the proportional fair (PF) scheduling algorithm [5], the users with their overall channel SNR near to the peaks are possibly scheduled and the system throughput is approaching to that of the coherent beamforming system.

However, in order to obtain the high throughput by the opportunistic beamforming, a large number of users must exist in each cell. In particular, as the number of transmit antennas of the base station increases, the number of required users grows rapidly. In [9], the conventional $\mathrm{OBF}$ is generalized by allowing multiple random weighting vectors at each time slot.

In the multiple weights OBF (MW-OBF) systems, there exist $Q$ mini-slots in each time slot. During each mini-slots, respectively, $Q$ known signals multiplied by $Q$ randomly selected independent weighting vectors $\boldsymbol{V}_{q}(t)(q=1, \ldots, Q)$ are transmitted. Then, during the $q$-th mini-slot, the overall channel gain is

$$
\tilde{H}_{q, k}(t)=\boldsymbol{V}_{q}(t) \boldsymbol{H}_{k}(t), q=1, \ldots, Q
$$

Each user measures its overall channel gain, $\left|\tilde{H}_{q, k}(t)\right|$, and feeds it back to the base station, then the base station determines the optimum weighting vector, $w^{o p t}(t)$, for data transmission and the selected user, $k^{*}$,

$$
\begin{gathered}
\left(k^{*}, q^{o p t}(t)\right)=\underset{q=1, . ., Q}{\arg \max _{k=1, \ldots, K}}\left(\max _{q, k}(t)\right) \\
w^{o p t}(t)=w_{q^{o p t}(t)}(t)
\end{gathered}
$$

where $R_{q, k}(t)$ is the transmitted rate for user $k$ if the $q$-th weight vector is used.

\section{New Scheme to Generate the Multiple Weights}

By allowing multiple random weighting vectors at each time slot, the throughput of the MW-OBF scheme is considerably improves compared to the conventional OBF since the employing the weights-selective diversity. However the using of several mini-slots will waste several radio resources and, thus, lower the spectrum efficiency.

In this section, a novel multiple weights generation method is developed by using only two mini-slots at each time slot. This novel scheme is illustrated with $N=2$.

Similar to the MW-OBF, two independent random vectors, $\boldsymbol{V}_{1}(t)=\boldsymbol{e}_{\theta}^{T}(t) \boldsymbol{\alpha}(t)$ and $\boldsymbol{V}_{2}(t)=\boldsymbol{e}_{\varphi}^{T}(t) \beta(t)$, are used at two mini-slots to create two equivalent channels, where $\boldsymbol{\alpha}=\operatorname{diag}\left(\alpha_{1}, \alpha_{2}\right), \boldsymbol{\beta}=\operatorname{diag}\left(\beta_{1}, \beta_{2}\right), \boldsymbol{e}_{\theta}^{T}=\left(e_{\theta 1}\right.$, $\left.e_{\theta 2}\right)^{T}, \boldsymbol{e}_{\varphi}^{T}=\left(e_{\varphi 1}, e_{\varphi 2}\right)^{T}$. And the two equivalent channels are, respectively,

$$
\begin{gathered}
\tilde{H}_{e q, k}^{(1)}(t)=\boldsymbol{V}_{1}(t) \boldsymbol{H}_{k}(t)=\boldsymbol{e}_{\theta}^{T}(t) \boldsymbol{\alpha}(t) \boldsymbol{H}_{k}(t) \\
\tilde{H}_{e q, k}^{(2)}(t)=\boldsymbol{V}_{2}(t) \boldsymbol{H}_{k}(t)=\boldsymbol{e}_{\varphi}^{T}(t) \boldsymbol{\beta}(t) \boldsymbol{H}_{k}(t)
\end{gathered}
$$

At the receiver, after the estimation of the two equivalent channels, linearly combining them as (the time variable $t$ is omitted for simplicity in the following),

$$
\begin{aligned}
\tilde{H}_{e q, k} & =\tilde{H}_{e q, k}^{(1)}+b \tilde{H}_{e q, k}^{(2)}=\boldsymbol{V}_{1} \boldsymbol{H}_{k}+b \boldsymbol{V}_{2} \boldsymbol{H}_{k} \\
& =\boldsymbol{e}_{\theta}^{T} \boldsymbol{\alpha} \boldsymbol{H}_{k}+b \boldsymbol{e}_{\varphi}^{T} \boldsymbol{\beta} \boldsymbol{H}_{k}=\left(\boldsymbol{e}_{\theta}^{T} \boldsymbol{\alpha}+b \boldsymbol{e}_{\varphi}^{T} \boldsymbol{\beta}\right) \boldsymbol{H}_{k}
\end{aligned}
$$

where $\boldsymbol{H}_{k}=\left[h_{1 k}, h_{2 k}\right]$, the complex value $b$ is the system parameter to be designed as followed.

Denoting

$$
\hat{\gamma}(b)=\boldsymbol{e}_{\theta}^{T} \boldsymbol{\alpha}+b \boldsymbol{e}_{\varphi}^{T} \boldsymbol{\beta}
$$

then $\tilde{H}_{e q, k}=\hat{\gamma}(b) \boldsymbol{H}_{k}$ can be viewed as the OBF channel using weighting factors $\hat{\gamma}(b)$. As in the conventional OBF, to preserve the total transmit power, $\hat{\gamma}(b)$ should be normalized as

$$
\gamma(b)=\hat{\gamma}(b) /|\hat{\gamma}(b)|
$$

Since $\hat{\gamma}(b)$ is the function of parameter $b$, selecting different $b$ can resulting in different multiple weighting vectors using only two mini-slots. Then the newly generated channel $\tilde{H}_{e q, k}$ is the linear combination of $\tilde{H}_{e q, k}^{(1)}$ and $\tilde{H}_{e q, k}^{(2)}$. Suppose that parameter $b$ is selected from a set with $W$ elements, then $W$ new channel can be generated.

In order not to increase the number of multiple operations, suppose $b$ is selected from the following set, $\{1,-1, j,-j\}$, with four elements (i.e., $W=4$ ). Then six weight vectors can be generated using only two minislots, thus improving the spectrum efficiency. Comparing with the original MW-OBF, the proposed scheme needs to estimate the equivalent channels at the two mini-time slots; however, this is easier than the quantized codebook scheme in [11] where channel gains from all users to each antenna must be estimated.

In the proposed scheme, users need feedback its maximum channel gain and the selected parameter $b$. Then transmitter schedules the users and calculating the current weights, using (6) and (7) based on the $b, V_{1}(t)$ 
and $\boldsymbol{V}_{2}(t)$.

\section{Numerical Results}

In this section, we present an extensive set of simulations to verify the effectiveness of the proposed scheme from different aspects. Firstly, since the achievable MUD gain in the system is determined by the dynamic range of the overall channel, which can be described by the probability density function (PDF) of the channels, our simulations depict the PDFs for different schemes. Then, if channels fade very slowly compared to the delay constraint of the application so that transmissions cannot wait until the channel reaches its peak, its QoS cannot be met. Therefore, the channel fluctuation speed, which can be described by the correlation function $(\mathrm{CF})$ of the overall channel, is simulated and given for different schemes. Finally the average throughput of the system for different schemes is simulated for comparison, using both maximum throughput (MAX) scheduling scheme and the PF scheduling scheme.

In the following simulations, we consider two transmit antennas at the base station under the Rician channel with different Rician factor $\kappa$ and average SNR $=0 \mathrm{~dB}$. We also suppose the availability of an error-free feedback channel from each user to the base station and the data rate achieved in each time slot is given by the Shannon limit.

\subsection{The PDFs and CFs of the Overall Channels}

To compare the performance of increasing the dynamic range of the equivalent channels, the PDFs of the chan- nels are plotted in Figure 1 for Rician channel (with $\kappa=10$ ) using different schemes, that is, none-OBF, $\mathrm{OBF}$, normal MW-OBF and the proposed scheme. The width of the PDF plot shows the dynamic range of the overall channel. From Figure 1, we can see that the dynamic range of the equivalent channels after $\mathrm{OBF}$ and the proposed scheme is much greater than that of the none-OBF, which ensures the larger obtainable MUD gain after OBF and the new MW-OBF scheme. Also comparing the proposed scheme with the normal MW$\mathrm{OBF}, \mathrm{OBF}$ and none-OBF, the probabilities that the overall channels have large amplitude are in descending order, which means that the proposed scheme has larger probability to approach high amplitude and, hence, the larger MUD gain.

If the maximum throughput scheduling scheme is employed at the transmitter, the user with the largest channel gain at a time slot will be scheduled to transmit data and the distribution of the peaks of the overall channels will be related to the system throughput directly. Hence, Figure 2 gives the PDFs of the channels' peak for none-OBF, OBF, normal MW-OBF and the proposed scheme, and 10 active users are in the system in the simulation. The four vertical bars, from left to right, indicate the mean values for the four schemes, respectively. The proposed scheme obtains the largest mean values and dynamic range among the four schemes.

Since the fluctuating speed within the time scale of interest is another source of the MUD gain, here we use the normalized correlation function (CF) of the overall channel as the indicator of the fluctuating speed, which is illustrated in Figure 3. And the Rician channels with $\kappa=30$ are employed in this simulation. For the same

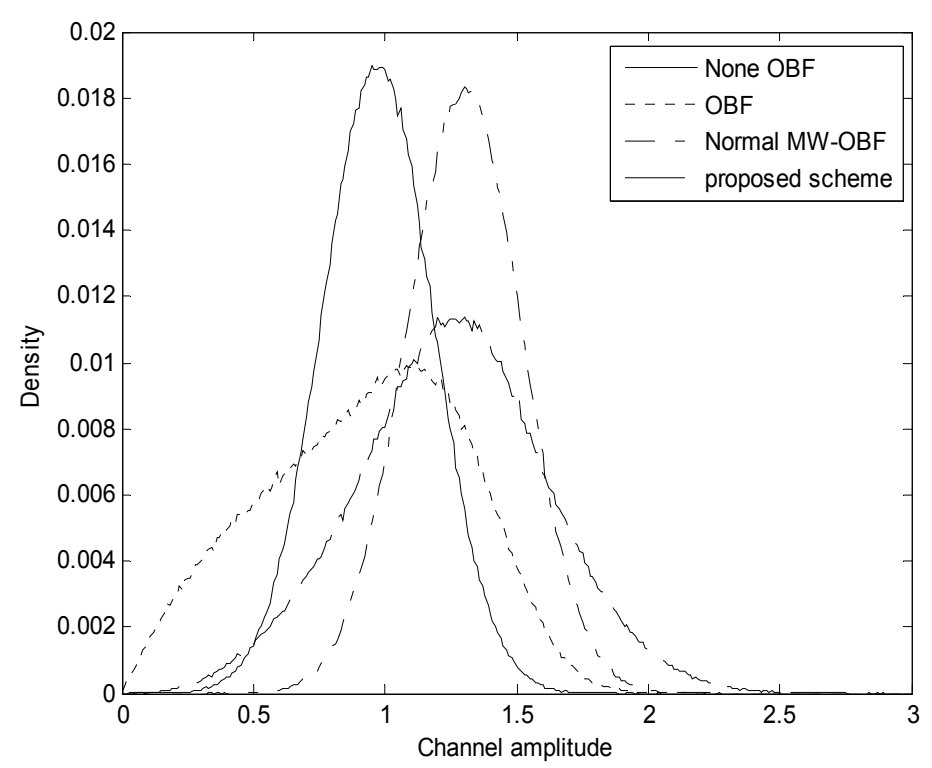

Figure 1. Channels PDFs for Rician channel. 


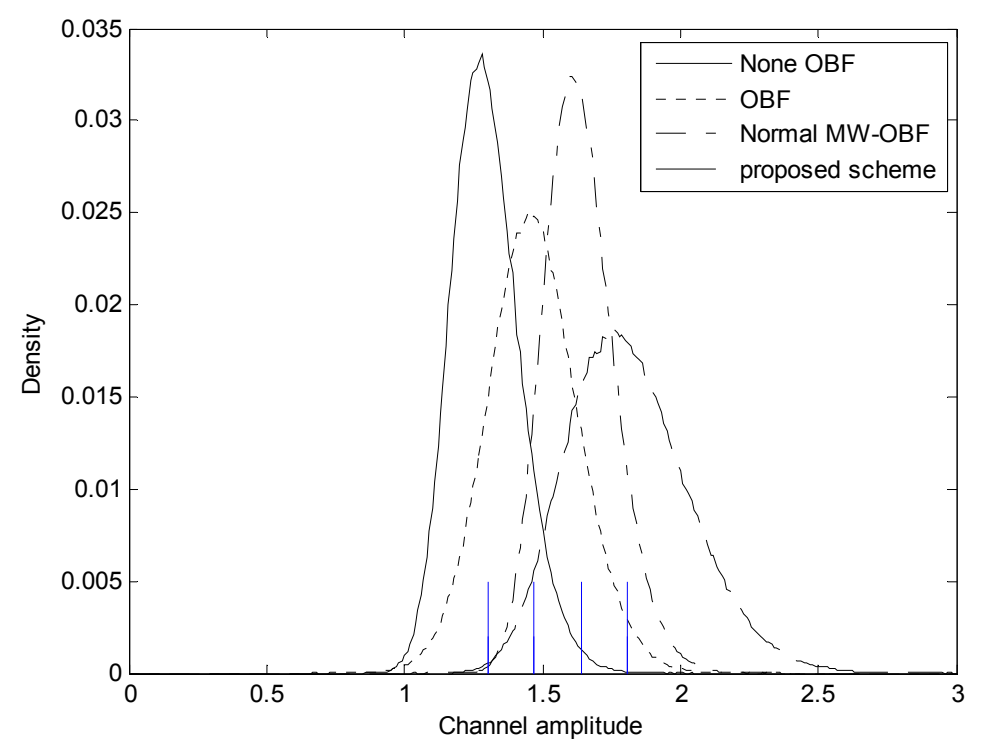

Figure 2. PDFs of channels' peak for Rician channel.

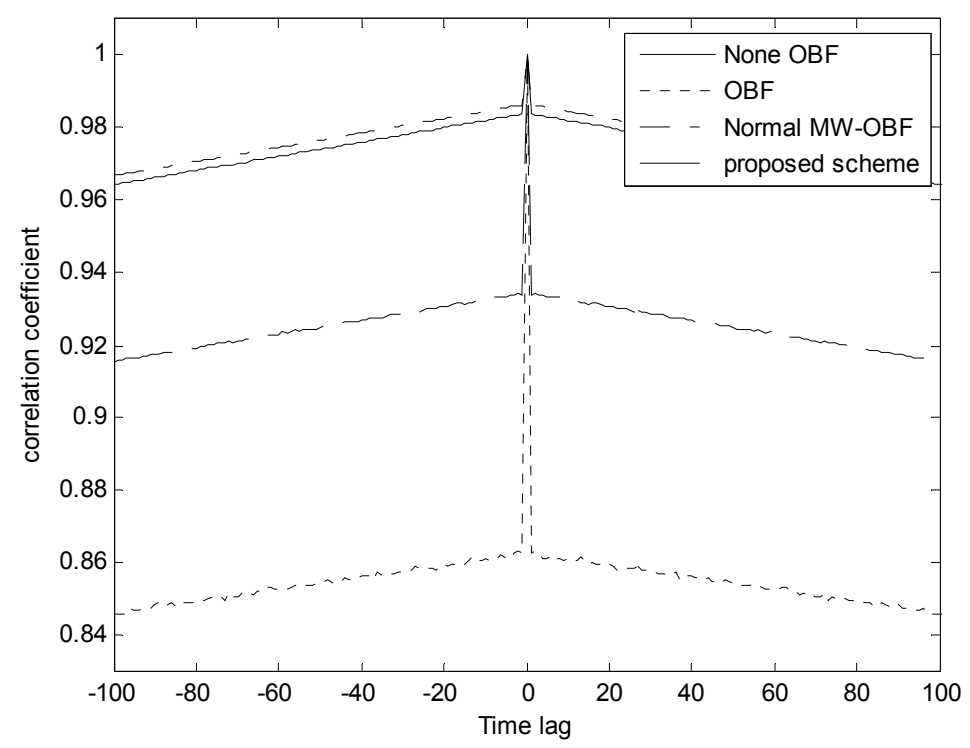

Figure 3. The normalized correlation function of the overall channel.

time lag, the larger the correlation coefficient is, the smaller the fluctuation speed is. So the proposed scheme has less correlation for same time lag, especially for small time lag compared to none-OBF and normal MW-OBF. Since the channels are generated via linearly combining the two equivalent channels, there is correlation among the channels generated in the proposed scheme. So comparing the proposed scheme with OBF, the correlation of the proposed scheme is larger than that of OBF. For example, when the time lag equals \pm 1 , the correlation coefficient of none-OBF, OBF, normal MW-OBF and the proposed scheme are $0.984,0.98,0.86$ and 0.925 , re- spectively.

From the above simulations, the resulting channels in the proposed scheme have larger dynamic range, larger probability to have high amplitudes, and larger fluctuating rate. We, therefore, can expect that the proposed scheme can obtain larger MUD gain, which will be illustrated in the following simulations.

\subsection{The System Average Throughput for Different Schemes}

The simulating parameters are same as those in [10]. The 


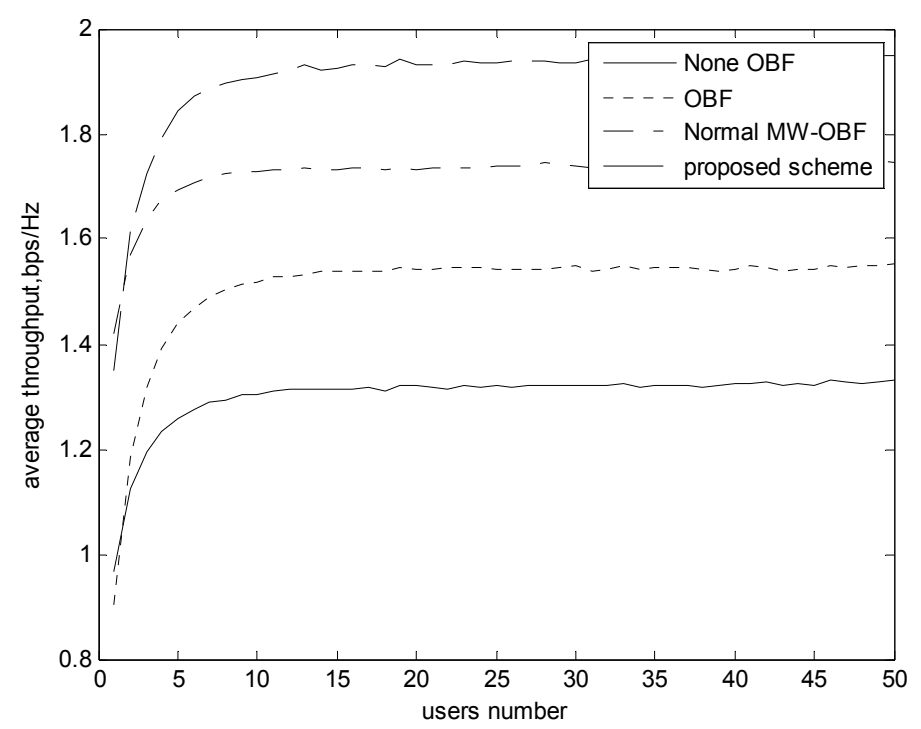

Figure 4. Average throughput using the PF scheme.

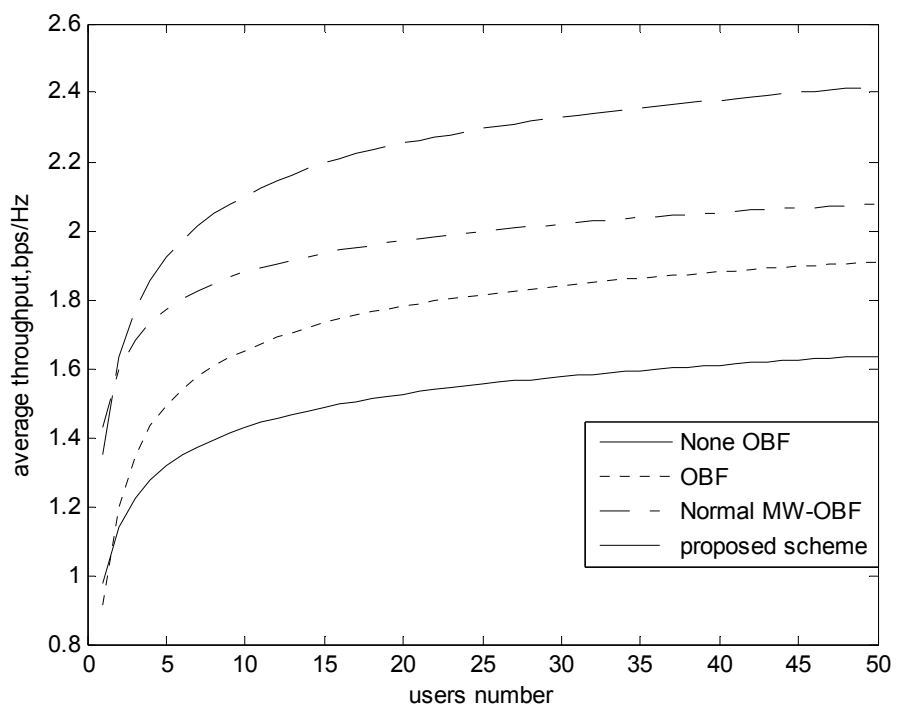

Figure 5. Average throughput using MAX scheduling scheme.

Rician channel with $\kappa=10$. Six mini-slots are used to generate six equivalent channels in MW-OBF, whereas two mini-slots are used in the proposed scheme to create two overall channels, and four additional channels are generated via linearly combining the available two overall channels.

Figures 4 and 5 illustrate the average throughput of the system using PF and MAX scheme for different schemes, respectively. The results show that, in both scheduling schemes, the average throughput are improved greatly, especially when the system with small number of users in MW-OBF and the proposed scheme. Meantime, the proposed scheme has larger throughput than MW-OBF.

\subsection{Throughput Variation with the Rician Factors}

Finally we study the performance variation of different schemes with the Rician factor $\kappa$, that is, to investigate the influence of the light of sight (LOS) on the system throughput. From the Figure 6 , it can be seen that with the increase of $\kappa$, the throughput for all scheme degrades because the throughput rely on the peak values of the instant overall channel. When the $\kappa$ factor increases, the channel fluctuations are reduced and the peak values of the instant overall channel are reduced, too. Compared with normal OBF, the proposed scheme can be improved the throughput, for example, for $\kappa=10$, 


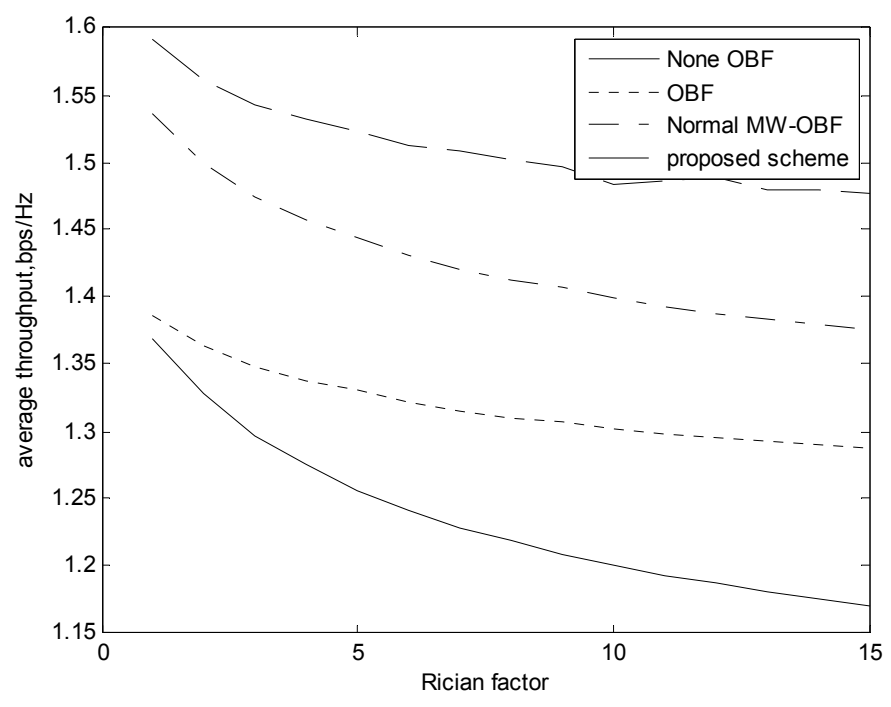

Figure 6. Throughput versus Rician factor of the channel.

more than $10 \%$ throughput enhancement can be obtained.

\section{Conclusions}

A new simple scheme to generate multiple weights used in opportunistic beamforming (OBF) system is proposed in this paper to deal with the performance degradation due to the fewer users in the OBF system. Only two mini-slots are employed to create effective channels, while more channel candidates can be obtained via linearly combining the two effective channels at the receiver side, thus increasing the multiuser diversity and the system throughputs. The simulation results show that the throughput can be improved using the proposed scheme.

\section{Acknowledgements}

This work is supported by Program for New Century Excellent Talents in University (NCET-08-0891), the Natural Science Foundation of China under the grant No. 60602053, and the Natural Science Foundation of Shaanxi Province under the grant No. 2007F02.

\section{References}

[1] E. Telatar, "Capacity of multi-antenna Gaussian channels," European Transactions on Telecommunications, Vol. 10, No. 6, pp. 585-596, 1999.

[2] F. Rashid-Farrokhi, K. J. R. LIU, and L. Tassiulas, "Transmit beamforming and power control for cellular wireless systems," IEEE Journal in Selected Areas on Communications, Vol. 16, No. 8, pp. 1437-1450, August 1998.

[3] M. Costa, "Writing on dirty paper," IEEE Transactions on Information Theory," Vol. 29, No. 3, pp. 439-441, May 1983.
[4] R. Knopp and P. A. Humblet, "Information capacity and power control in single cell multiuser communications," In Proceedings of IEEE International Conference on Communications, pp. 331-335, 1995.

[5] P. Viswanath, D. N. C. Tse, and R. Laroia, "Opportunistic beamforming using dumb antennas," IEEE Transactions on Information Theory, Vol. 48, No. 6 pp. 1277 1294, June 2002.

[6] M. Sharif and B. Hassibi, "On the capacity of MIMO broadcast channels with partial side information," IEEE Transactions on Information Theory, Vol. 51, No. 2, pp. 506-522, February 2005.

[7] W. Zhang and K. B. Letaief, "MIMO broadcast scheduling with limited feedback," IEEE Journal in Selected Areas on Communications, Vol. 25, No. 7, pp. 1457-1467, July 2007.

[8] M. Kountouris and D. Gesbert, "Memory-based opportunistic multi-user beamforming," In Proceedings of International Symposium on Information Theory, pp. 1426-1430, September 2005.

[9] I. R. Baran and B. F. Uchoa-Filho, "Enhanced opportunistic beamforming for Jakes-correlated fading channels," In Proceedings of International Telecommunications Symposium, pp. 1024-1029, Fortaleza, Ceara, September 2006.

[10] II-M. Kim, S. C. Hong, and S. S. Ghassemzadeh, "Opportunistic beamforming based on multiple weighting vectors," IEEE Transactions on Wireless Communications, Vol. 4, No. 6, pp. 2683-2687, November 2005.

[11] M. Zeng, J. Wang, and S. Q. Li, "Rate upper bound and optimal number of weight vectors for opportunistic beamforming," In proceedings of IEEE Vehicular Technology Conference, Fall, pp. 661-665, September 30 2007-October 3, 2007.

[12] J. Kang, I. K. Choi, D. S. Kwon, and C. Y. Lee, "An opportunistic beamforming technique using a quantized codebook," In proceedings of IEEE Vehicular Technology Conference, pp. 1647-1651, Spring, 2007. 